\title{
Scarlet Fever in an Adult
}

\author{
Satoshi Kutsuna, Kayoko Hayakawa and Norio Ohmagari
}

Key words: scarlet fever, Streptococcus pyogenes, pharyngitis, strawberry-red tongue, desquamation

(Intern Med 53: 167-168, 2014)

(DOI: 10.2169/internalmedicine.53.1486)

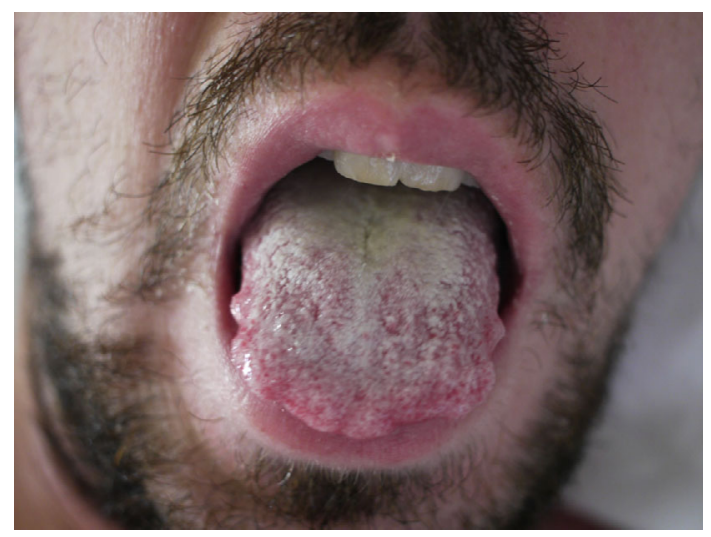

Picture 1.

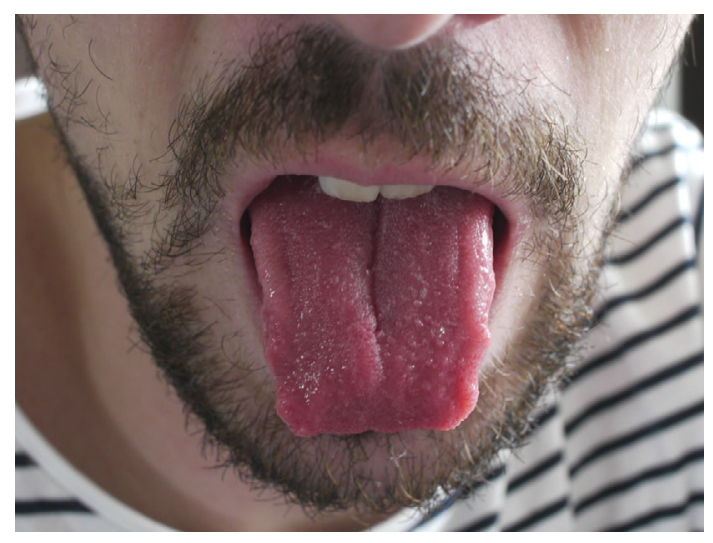

Picture 3.

A 29-year-old French man living in Tokyo presented to our emergency department with a fever, sore throat and rash. His tongue had a white coating (Picture 1), and diffuse erythema (Picture 2) that blanched on pressure was noted over the neck and trunk. A rapid streptococcal antigen test of the pharynx was positive; thus, treatment with penicillin was initiated. On the third day of treatment, the white coating on the patient's tongue disappeared and the characteris-

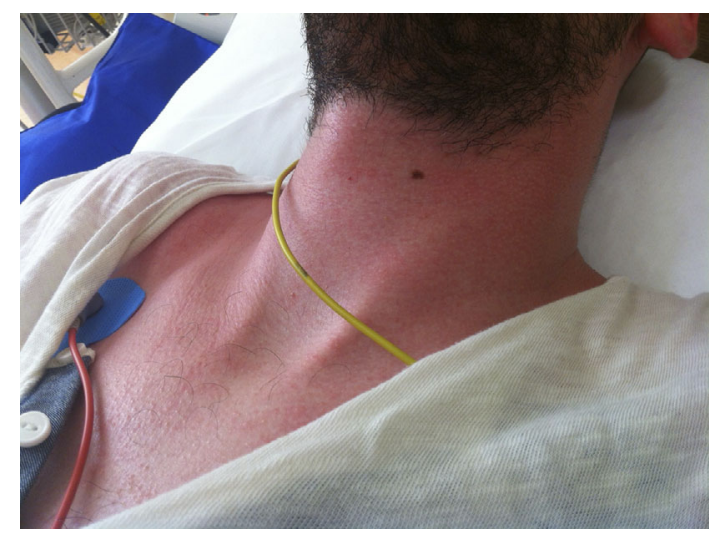

Picture 2.

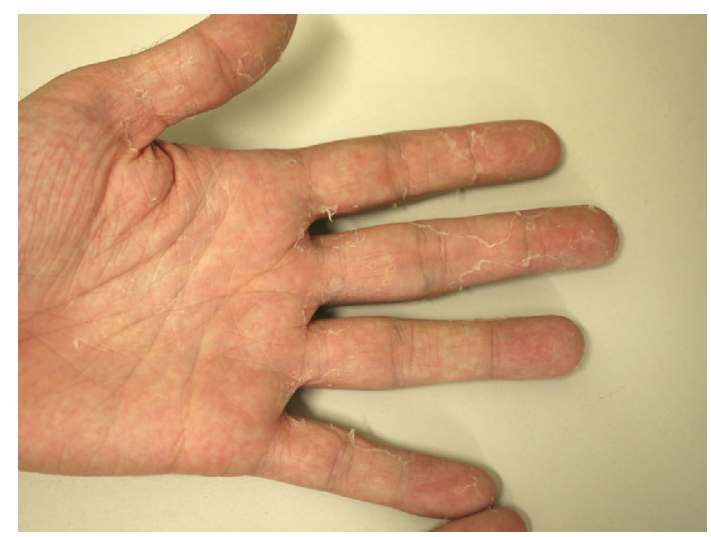

Picture 4.

tic strawberry-red tongue appeared (Picture 3). A throat culture became positive for Streptococcus pyogenes. The patient was diagnosed with scarlet fever and completed a 10day course of treatment. The rash eventually desquamated (Picture 4). Scarlet fever is a pediatric disease, as most of the population acquires the antitoxin when they are affected by Streptococcus, and is rarely seen in adults (1). Even in adult patients, physicians should be aware of the characteris- 
tic appearance of a strawberry-red tongue that is initially covered with a white coat (2).

The authors state that they have no Conflict of Interest (COI).

\section{References}

1. Davies RJ, de Bono JP. A young rash on old shoulders-scarlet fever in an adult male. Lancet Infect Dis 2: 750, 2002.

2. Bisno AL, Stevens DL. Streptococcus pyogenes. In: Mandell, Douglas, and Bennett's Principles and Practice of Infectious Diseases. 7th ed. Mandell GL, Bennett JE, Dolin R, Eds. Churchill Livingstone, Philadelphia, 2009: 2593-2610.

(C) 2014 The Japanese Society of Internal Medicine http://www.naika.or.jp/imonline/index.html 